\title{
Capsule endoscopy of the small bowel
}

\author{
Mark E. McAlindon ${ }^{1}$, Hey-Long Ching ${ }^{1}$, Diana Yung ${ }^{2}$, Reena Sidhu ${ }^{1}$, Anastasios Koulaouzidis ${ }^{2}$ \\ ${ }^{1}$ Gastroenterology \& Liver Unit, Royal Hallamshire Hospital, Sheffield, UK; ${ }^{2}$ Endoscopy Unit, The Royal Infirmary of Edinburgh, Edinburgh, UK \\ Contributions: (I) Conception and design: All authors; (II) Administrative support: None; (III) Provision of study materials or patients: None; (IV) \\ Collection and assembly of data: None; (V) Data analysis and interpretation: None; (VI) Manuscript writing: All authors; (VII) Final approval of \\ manuscript: All authors. \\ Correspondence to: Mark E. McAlindon, BMedSci, BMBS, MRCP, DM. Consultant Gastroenterologist, Room P39, P Floor, Department of \\ Gastroenterology, Royal Hallamshire Hospital, Glossop Road, S10 2JF, Sheffield, UK. Email: mark.mcalindon@sth.nhs.uk.
}

\begin{abstract}
Capsule endoscopy (CE) is a first line small bowel investigative modality which provides more sensitive mucosal imaging than comparators. It is a non-invasive, non-irradiating tool well tolerated by patients. The risk of retention of the capsule can be minimised by ensuring luminal patency using the Agile patency device. Research continues into how to minimise missed pathology and variability in the identification of pathology or interpretation of images. The consensus is that bowel preparation using laxatives improves visibility and diagnostic yield. Research includes the development of image recognition software, both to eliminate sequentially identical images to improve viewing speed and to select or enhance images likely to represent pathology. However, careful reading by experienced capsule endoscopists remains the benchmark. This should be performed at a speed comfortable to the viewer, probably at a maximum of 15 frames per second. Some prior experience of endoscopy appears to be helpful for novice capsule endoscopists and formal training on a hands-on training course seems to improve pathology recognition, for novices and for those with CE experience.
\end{abstract}

Keywords: Capsule endoscopy (CE); small bowel; software; indication; reading; complications

Submitted Aug 17, 2016. Accepted for publication Aug 24, 2016.

doi: $10.21037 /$ atm.2016.09.18

View this article at: http://dx.doi.org/10.21037/atm.2016.09.18

\section{Development of capsule endoscopy (CE)}

The CE is a swallowable pill camera which contains light emitting diodes and a battery powered imaging device. This is a complementary metal oxide semi-conductor (PillCam SB3, Medtronic, Minneapolis, USA) or a charge-coupled device (MiroCam, IntroMedic, Seoul, Korea; OMOM capsule, Jinshan Science and Technology, Chongqing, China; EndoCapsule, Olympus, Tokyo, Japan). Most models transmit images using radiofrequency to an external data recorder, an energy consuming process. This initial limited image capture time to 8 hours, although the PillCam SB3 now acquires images for up to 12 hours. The MiroCam saves energy by using electric field propagation, in which the human body is used as a semiconductor for data transmission, thus extending battery life to over 12 hours and the CapsoCam (CapsoVision, Saratoga, USA) does so by retaining data on a USB device, the capsule requiring retrieval for data download onto a computer yielding 16 hours of images. The latter is the only capsule which views the mucosa from the side of the device, from which four cameras, each with a $90^{\circ}$ view, provide a $360^{\circ}$ circumferential image (1). Once downloaded, images can be viewed at enhanced speed on a computer monitor in video format and reported by the endoscopist in 30-60 minutes.

Uncoupling the imaging device from the monitor screen rendered the need for the optical cabling used in conventional endoscopy obsolete and allowed a completely remote method of visualising the intestinal mucosa, noninvasive and much better tolerated by patients $(2,3)$. CE was first designed with the aim of investigating the small bowel, hitherto very difficult to access with an endoscope, and small in diameter, such that a capsule of just over a centimeter in diameter would pass through the lumen under the action of peristalsis in a relatively stable longitudinal direction of travel to the caecum. 


\section{Indications for small bowel CE}

CE has rapidly become a first line small bowel investigative modality, improving diagnostic yield in patients with small bowel bleeding when compared to push enteroscopy, small bowel barium contrast radiology, computed tomography and magnetic resonance (MR) imaging (4). It is also more sensitive in detecting mucosal inflammation in patients with suspected Crohn's disease than the comparators, although has a complementary role to MR in patients with established Crohn's disease when fibrosing or penetrating disease is suspected and cross sectional images are required (5). It is not commonly used to diagnose coeliac disease (except perhaps in those with positive antibody serology who refuse to undergo conventional upper gastrointestinal endoscopy for duodenal biopsies) as the sensitivity is only $89 \%$, villous atrophy being absent or mild only (grade $3 \mathrm{~A}$ ) in the early Marsh grades of coeliac disease and therefore impossible or difficult to recognize even though mucosal views obtained are at 8 -fold magnification (6). It may help in distinguishing the different causes of villous atrophy, particularly in endomysial antibody negative patients, and identifies features of type II refractory coeliac disease in those not responding, or losing response, to a gluten free diet $(7,8)$. CE probably identifies more small polyps in Peutz-Jeghers syndrome than MR enterography or enteroclysis, but this adds little clinical benefit as only larger polyps (of over $1.5 \mathrm{~cm}$ ) are resected $(9,10)$. Although most authorities do not regard recurrent abdominal pain as a strong indication for CE, meta-analysis (albeit of studies of what are likely to represent select cohorts of patients with particularly troublesome symptoms) suggests a diagnostic yield as high as $21 \%$ (11). Cheifetz and Lewis describe using CE when recurrent small bowel obstruction was strongly suspected, but which was not confirmed by radiology: diagnoses were made in 5 of 19 patients, 4 of whom retained capsules but in whom surgery resolved symptoms (12).

\section{Risks of small bowel CE}

Retention of the capsule behind a stricture is the main risk of CE. Systematic review of over 20,000 procedures suggests that it occurs in $1.2 \%$ of patients being investigated for anaemia, in $2.6 \%$ of those known or suspected to have Crohn's disease, increasing (as discussed earlier) to $21 \%$ of patients with suspected obstruction $(12,13)$. In addition to Crohn's disease, factors thought to increase the risk of retention are long term (perhaps at least 6 months) use of non-steroidal anti-inflammatory drugs and previous abdominopelvic radiation therapy $(13,14)$. Retained capsules very rarely cause obstruction, strictures being irregular in shape and therefore unlikely to accommodate the round dome of the device unless simultaneously plugged with residual undigested food material. Of course, capsule retention would not usually be regarded as a complication if caused by conditions requiring surgical treatment, such as tumors. Retention in patients with Crohn's disease, however, may complicate management as surgery is not usually first line treatment. Capsules may pass after a prolonged period of retention (for example when oedema diminishes after the treatment of Crohn's disease or the cessation of non-steroidal anti-inflammatory drugs) and have been retained for 6 years or more without causing problems (15). Unfortunately, small bowel radiology (barium, CT or MR) is not wholly reliable in excluding strictures (14), hence the development of the Agile patency device (Medtronic). This is a capsule the same size and shape as the PillCam SB3 in which barium, lactose and a radiofrequency tag are contained in an outer membrane. Wax plugs at either end of the device dissolve in intestinal contents over time allowing the barium to escape and the membrane to crumple and pass through the stricture. Studies suggest that if the handheld radiofrequency scanner fails to detect the signal 30 hours after ingestion, implying that the patient has passed the device, or if the patient sees it excreted intact subsequently, CE can be performed safely. Half the patients swallowing Agile capsules do not pass them within 30 hours, however, and whilst the barium content allows easy identification, plain abdominal radiographs are insufficient to allow reliable localisation of the device. In the event that it has not been passed at 30 hours, a scout film to target a few CT slices through the identified Agile capsule provides a single step, reliable, low radiation protocol to confirm retention in the small bowel (often with a clue as to the pathology causing it) or safe passage into the colon (16).

\section{Optimising small bowel CE}

The ideal endoscopy should be well tolerated, easy to perform and provide diagnostic information quickly and accurately. Therefore, optimising performance might be considered at each of three stages of the procedural pathway: preparing the bowel in advance of the procedure, the development of software to improve sensitivity of pathology detection whilst minimizing video reading time and improvement of the endoscopists' reading and 
interpretation.

\section{Bowel preparation}

Many studies have addressed the issues of cleansing the bowel of material which hampers small bowel mucosal views: systematic and meta-analyses suggest that preprocedural laxatives improve both visibility and diagnostic yield without affecting transit times and the administration of simethicone may reduce the presence of bubbles which obscure views. Promotility agents do not improve diagnostic yield, although metoclopramide may be used to enhance gastric emptying in patients in whom the capsule does not enter the small bowel quickly (usually when a real time viewer attached to the data recorder still shows gastric mucosa 30-60 min after ingestion) (17-23).

\section{CE reading software}

Although CE is a different tool, conventional endoscopy will always be seen as a benchmark. Therefore 30-60 minutes to read and report on a CE video may be seen as unduly long. Maintaining a high level of concentration whilst viewing 50-100,000 images of the same organ is difficult, particularly as this form of endoscopy is completely passive, with no additional stimulus provided by the need to manipulate an instrument or interact with patient or other staff. Therefore, it seems possible that reports of missed pathology (24-27) and significant interpersonal variability in reporting (28-30) stems in part from operator failure and reflects the difficulty in sustaining focus for this length of time.

These concerns (which apply to all forms of endoscopy to some extent), have long been recognised and much has been done to try and address them. 'Automatic' or 'express view' modes (in which in-built software selects out sequentially identical images) seem to allow a reduced reading time without having an impact on diagnostic yield. The video can be run as a single, double (consecutive images seen side by side) or quadruple (four consecutive images seen simultaneously) view. Video speed can be increased, although it appears that the miss rate may increase with greater viewing speeds (and an optimal viewing speed of no more than 15 frames per second has been recommended) although dual or quadruple view may allow quicker reading times without obvious diminution of pathology detection. The QuickView facility on the Medtronic system (other systems have similar softwares) selects $10 \%$ (the proportion selected can be altered by the operator) of the most standout images for viewing, and earlier iterations of this software suggest a sensitivity of lesion detection of $89-100 \%$ compared to conventional viewing with a significant reduction in reading time (28,29,31-40). Some operators use it as a preliminary screen to look for obvious pathology if information is needed quickly. The spontaneous blood indicator selects images containing multiple red pixels, potentially useful in detecting bleeding lesions, but has variable reported sensitivities and specificities for detecting potential bleeding lesions or active bleeding of only $26-81 \%$ and $33-76 \%$ respectively $(28,29,39-43)$.

The holy grail of software development remains the automated detection of frames containing pathology (44-46). It is hoped that computational methods will be developed to enhance diagnostic yield. However, incorporating machine learning algorithms into CE reading is difficult as large amounts of image annotations are required to develop image recognition software. One limitation of the current databases is the lack of graphic annotations of pathologies

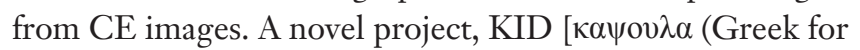
'capsule') interactive database], aims to provide a reference database for the research and development of these machine learning algorithms (47).

Virtual chromoendoscopy has been applied to CE, as with conventional endoscopy, with different systems allowing images to be viewed under different Fujinon intelligent colour enhancement (FICE) settings as well as standard white light. As highlighted by Spada et al. (48), the hope was that this would lead to an improvement in CE lesion detection rate and better clarity of lesions enabling their characterisation and determination of their significance. There is some suggestion that FICE might help to distinguish subtle abnormalities from artefact (49), but a recent meta-analysis by the co-authors suggest that virtual chromoendoscopy does not improve diagnostic yield, nor allow better definition of lesions (Koulaouzidis, personal communication). This is consistent with the recent American Society of Gastroenterology technical report about FICE in conventional endoscopy, which concluded that there was currently no evidence to support an optimal FICE mode for tissue diagnosis and differentiation (50).

Other virtual chromoendoscopy software for CE exists, such as blue mode and Augmented Live-body Image Color-spectrum Enhancement (ALICE) (IntroMedic, Seoul, Korea). Several studies of reading in blue mode, however, have failed to demonstrate any clear benefit $(40,51,52)$. There is only one published study using 


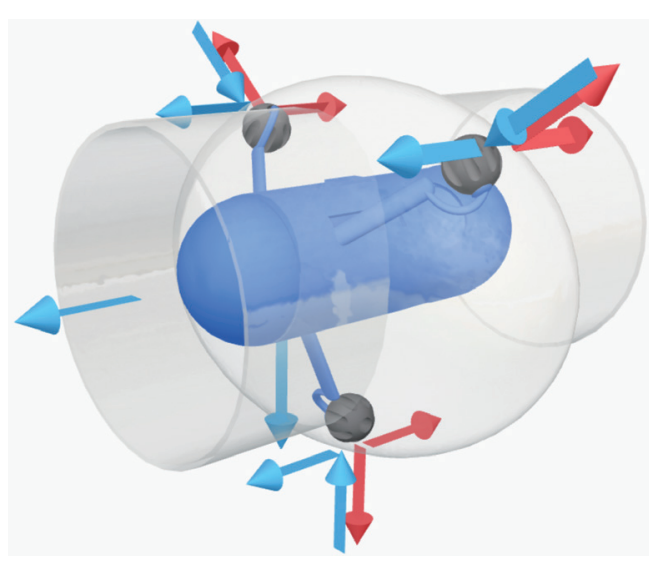

Figure 1 The OdoCapsule: extended legs stabilize the capsule during transit and rotating wheels measure distance travelled.

ALICE, an abstract which reported improvement in the visibility of flat and depressed small bowel lesions, so potential benefits of this chromoendoscopy technique remain uncertain (53). In 2011, Aihara et al. investigated a 'contrast capsule' equipped with a white light lightemitting diode selected to give increased illumination intensity of blue light following which the contrast images were developed by extracting only green and blue light data, producing dark green vascular structures (54). Further research is needed to determine whether this translates to any clinical benefit.

A perceived limitation of $\mathrm{CE}$ is the lack of reliable pathology localization. Current systems give an approximate localization based on the time taken for the capsule to travel from the first duodenal image to the pathology and on to the caecum (the duodenum and caecum being easily identifiable landmarks). The Medtronic system recognises that the capsule does not pass at a uniform speed through the gut and additionally provides information on the speed of travel at any point, by measuring the degree of change between successive images (the progress indicator). Several systems provide a two-dimensional image of a linear route map of the travel path of a radiofrequency-emitting capsule by triangulating signals detected by external sensors attached to different fixed sites on the anterior abdominal wall (55). An interesting study using radiofrequency triangulation around the abdominal wall to provide three-dimensional localization showed an average spatial error of $13.26 \mathrm{~cm}^{3}$ when compared with plain radiography (anteroposterior and lateral abdominal radiographs) (56). The 'OdoCapsule' represents another approach which aims not only to provide localization data, but also stabilises capsule transit by reducing side to side and tumbling movements (Figure 1). The capsule contains three extendable legs with rotating wheels at their extremities which span the cross sectional area of the lumen. Ex vivo animal studies suggest that this model might be able to act as an odometer, measuring distance travelled by wheel rotation, as well as providing stability in transit. The latter feature would be a valuable asset to those pursuing the development of computer image recognition systems. These rely on identifying matching features in sequential images which can be assembled into a complete picture of a lesion: rapid and unpredictable capsule movement is more likely to produce sequential images which are very different and less likely to include recognizable features or mapping points $(57,58)$. Further recent research has considered active magnetic localisation methods, MR, ultrasound and positron emission imagingbased approaches (58). However, although none of these methods are accurate or reliable as yet, exact localization of a lesion is not always essential: for the purposes of deviceassisted enteroscopy or surgery, it may be sufficient to know if the pathology is in the duodenum (in which surgery may be more complex), or otherwise if the lesion is proximal or distal, which may affect the route of insertion (antegrade or retrograde) of a device-assisted enteroscope (14).

\section{Reading and reporting CE videos}

Numerous studies have compared CE reading between experts, novices and nurses (59-61). Whilst some level of prior conventional endoscopy experience is probably helpful for novice CE readers in training $(59,62)$, a high level of experience does not seem to add further benefit (63). Nurses are as capable of reading and recognising CE abnormalities as doctors: whilst they may identify additional insignificant findings, no serious pathology is missed compared to expert physicians $(60,61,64)$. There are currently several CE training courses established in Europe. A simple evaluation tool (ET-CET) based on interpretation of video clips was carried out to identify factors related to the learning curve of $\mathrm{CE}$ among trainees, nurses and doctors attending courses offering a common core curriculum (62). The study found that previous $\mathrm{CE}$ experience was associated with higher baseline scores (before the course) for correct diagnosis. There was also a plateau in baseline scores for those who attended having an experience of more than 25 prior CE readings. This would support the benefits of hands-on training courses at this early stage, as further improvement 


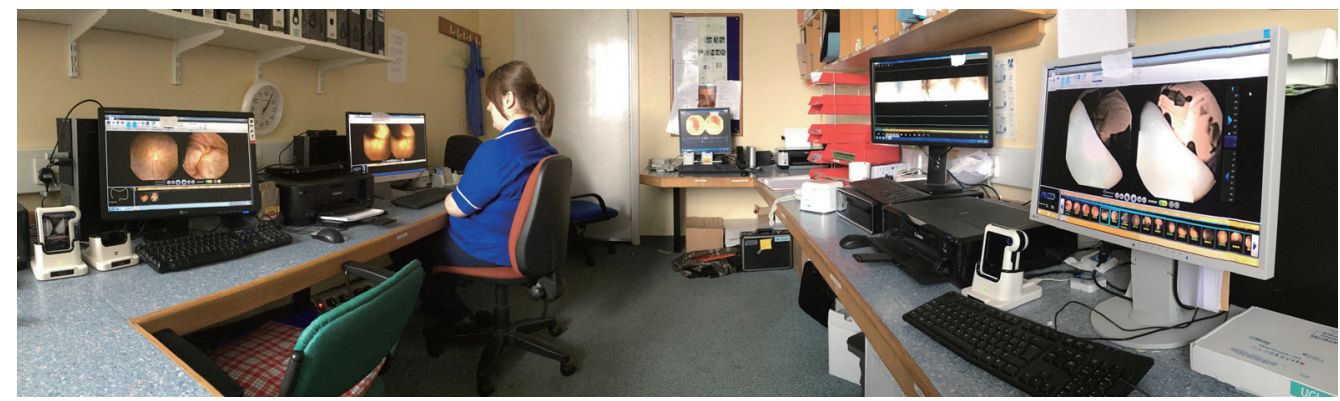

Figure 2 The gastrointestinal diagnostic hub: video data downloaded following capsule endoscopy in the community, read, reported and returned with advice to the referring clinician.

was demonstrated in all, irrespective of prior experience and furthermore, improvement was not dependent on profession, CE system or course setting. Recommended training for $\mathrm{CE}$ is based on societal guidelines and expert opinion whilst formal credentialing is still being developed in many countries (63). In contrast to standard endoscopy indicators which focus predominantly on technical competence, CE requires a more cognitive skill set based more on observation and interpretation of significant findings from computer images and providing appropriate management advice.

\section{The end of conventional endoscopy as a diagnostic tool?}

The software and technology behind CE is evolving rapidly and there is a sense that a sensitive, non-invasive investigative method for the whole gastrointestinal tract is within reach. Image quality, capture rates and battery life have rapidly improved, models to visualize the upper gastrointestinal tract and colon are available and research programmes are actively investigating image recognition software to improve pathology detection, reduce reliance on the human eye and speed up the diagnostic process. It is already possible for patients to have CE procedures in the community or at home. In the near future it may be that the data acquired is uploaded to gastrointestinal diagnostic hubs for analysis and interpretation, the endoscopy rooms of the future (Figure 2). These tools will identify the minority of patients who need conventional (intubational) endoscopy to obtain biopsies for histology or therapeutic intervention. Although it was as long ago as 2000 when first suggested by Iddan et al., "the discomfort of internal gastrointestinal examination may soon be a thing of the past" (65).

\section{Acknowledgements}

None.

\section{Footnote}

Conflicts of Interest: ME McAlindon has received honoraria from IntroMedic Ltd and CapsoVision, travel support from Given Imaging Ltd and research grant support from Given Imaging Ltd and SynMed UK. A Koulaouzidis: honoraria from \& member of advisory board(s) for Dr. Falk Pharma UK, grant form Given Imaging/ESGE, material support for research from SynMed UK, travel support from Almirall \& Dr. Falk Pharma. D Yung: grant from Core/Dr. Falk and travel support from Dr. Falk Pharma UK. The other authors have no conflicts of interest to declare.

\section{References}

1. Li Z, Carter D, Eliakim R, et al. The current main types of capsule endoscopy. In: Li ZS, Liao Z, McAlindon M. editors. Handbook of Capsule Endoscopy. Dordrecht: Springer, 2014.

2. Lapalus MG, Dumortier J, Fumex F, et al. Esophageal capsule endoscopy versus esophagogastroduodenoscopy for evaluating portal hypertension: a prospective comparative study of performance and tolerance. Endoscopy 2006;38:36-41.

3. Irvine AJ, Sanders DS, Hopper AD, et al. How does tolerability of double balloon enteroscopy compare to other forms of endoscopy? Frontline Gastroenterology 2016;7:41-6.

4. Triester SL, Leighton JA, Leontiadis GI, et al. A metaanalysis of the yield of capsule endoscopy compared to other diagnostic modalities in patients with 
obscure gastrointestinal bleeding. Am J Gastroenterol 2005;100:2407-18.

5. Dionisio PM, Gurudu SR, Leighton JA, et al. Capsule endoscopy has a significantly higher diagnostic yield in patients with suspected and established small-bowel Crohn's disease: a meta-analysis. Am J Gastroenterol 2010;105:1240-8; quiz 1249.

6. Rokkas T, Niv Y. The role of video capsule endoscopy in the diagnosis of celiac disease: a meta-analysis. Eur J Gastroenterol Hepatol 2012;24:303-8.

7. Kurien M, Evans KE, Aziz I, et al. Capsule endoscopy in adult celiac disease: a potential role in equivocal cases of celiac disease? Gastrointest Endosc 2013;77:227-32.

8. Barret M, Malamut G, Rahmi G, et al. Diagnostic yield of capsule endoscopy in refractory celiac disease. Am J Gastroenterol 2012;107:1546-53.

9. Gupta A, Postgate AJ, Burling D, et al. A prospective study of MR enterography versus capsule endoscopy for the surveillance of adult patients with Peutz-Jeghers syndrome. AJR Am J Roentgenol 2010;195:108-16.

10. Urquhart P, Grimpen F, Lim GJ, et al. Capsule endoscopy versus magnetic resonance enterography for the detection of small bowel polyps in Peutz-Jeghers syndrome. Fam Cancer 2014;13:249-55.

11. Xue M, Chen X, Shi L, et al. Small-bowel capsule endoscopy in patients with unexplained chronic abdominal pain: a systematic review. Gastrointest Endosc 2015;81:186-93.

12. Cheifetz AS, Lewis BS. Capsule endoscopy retention: is it a complication? J Clin Gastroenterol 2006;40:688-91.

13. Liao Z, Gao R, Xu C, et al. Indications and detection, completion, and retention rates of small-bowel capsule endoscopy: a systematic review. Gastrointest Endosc 2010;71:280-6.

14. Sidhu R, Sanders DS, Morris AJ, et al. Guidelines on small bowel enteroscopy and capsule endoscopy in adults. Gut 2008;57:125-36.

15. Harrington $\mathrm{C}$, Rodgers $\mathrm{C}$. The longest duration of retention of a video capsule. BMJ Case Rep 2014;2014.

16. Assadsangabi A, Blakeborough A, Drew K, et al. Small bowel patency assessment using the patency device and a novel targeted (limited radiation) computed tomographybased protocol. J Gastroenterol Hepatol 2015;30:984-9.

17. Niv Y. Efficiency of bowel preparation for capsule endoscopy examination: a meta-analysis. World J Gastroenterol 2008;14:1313-7.

18. Rokkas T, Papaxoinis K, Triantafyllou K, et al. Does purgative preparation influence the diagnostic yield of small bowel video capsule endoscopy?: A meta-analysis. Am J Gastroenterol 2009;104:219-27.

19. Belsey J, Crosta C, Epstein O, et al. Meta-analysis: efficacy of small bowel preparation for small bowel video capsule endoscopy. Curr Med Res Opin 2012;28:1883-90.

20. Kotwal VS, Attar BM, Gupta S, et al. Should bowel preparation, antifoaming agents, or prokinetics be used before video capsule endoscopy? A systematic review and meta-analysis. Eur J Gastroenterol Hepatol 2014;26:137-45.

21. Koulaouzidis A, Giannakou A, Yung DE, et al. Do prokinetics influence the completion rate in small-bowel capsule endoscopy? A systematic review and meta-analysis. Curr Med Res Opin 2013;29:1171-85.

22. Zhang JS, Ye LP, Zhang JL, et al. Intramuscular injection of metoclopramide decreases the gastric transit time and does not increase the complete examination rate of capsule endoscopy: a prospective randomized controlled trial. Hepatogastroenterology 2011;58:1618-21.

23. Sidhu R, Drew K, Sanders DS, et al. Does the selective use of metoclopramide improve the completion rate of small-bowel capsule endoscopy? Gastrointest Endosc 2010;72:670-1; author reply 671.

24. Ross A, Mehdizadeh S, Tokar J, et al. Double balloon enteroscopy detects small bowel mass lesions missed by capsule endoscopy. Dig Dis Sci 2008;53:2140-3.

25. Postgate A, Despott E, Burling D, et al. Significant smallbowel lesions detected by alternative diagnostic modalities after negative capsule endoscopy. Gastrointest Endosc 2008;68:1209-14.

26. Hakim FA, Alexander JA, Huprich JE, et al. CTenterography may identify small bowel tumors not detected by capsule endoscopy: eight years experience at Mayo Clinic Rochester. Dig Dis Sci 2011;56:2914-9.

27. Zagorowicz ES, Pietrzak AM, Wronska E, et al. Small bowel tumors detected and missed during capsule endoscopy: single center experience. World J Gastroenterol 2013;19:9043-8.

28. Rondonotti E, Soncini M, Girelli CM, et al. Can we improve the detection rate and interobserver agreement in capsule endoscopy? Dig Liver Dis 2012;44:1006-11.

29. Zheng Y, Hawkins L, Wolff J, et al. Detection of lesions during capsule endoscopy: physician performance is disappointing. Am J Gastroenterol 2012;107:554-60.

30. Pezzoli A, Cannizzaro R, Pennazio M, et al. Interobserver agreement in describing video capsule endoscopy findings: a multicentre prospective study. Dig Liver Dis 2011;43:126-31.

31. Kyriakos N, Karagiannis S, Galanis P, et al. Evaluation of 
four time-saving methods of reading capsule endoscopy videos. Eur J Gastroenterol Hepatol 2012;24:1276-80.

32. Nakamura M, Murino A, O'Rourke A, et al. A critical analysis of the effect of view mode and frame rate on reading time and lesion detection during capsule endoscopy. Dig Dis Sci 2015;60:1743-7.

33. Shiotani A, Honda K, Kawakami M, et al. Analysis of small-bowel capsule endoscopy reading by using Quickview mode: training assistants for reading may produce a high diagnostic yield and save time for physicians. J Clin Gastroenterol 2012;46:e92-5.

34. Subramanian V, Mannath J, Telakis E, et al. Efficacy of new playback functions at reducing small-bowel wireless capsule endoscopy reading times. Dig Dis Sci 2012;57:1624-8.

35. Saurin JC, Lapalus MG, Cholet F, et al. Can we shorten the small-bowel capsule reading time with the "Quick-view" image detection system? Dig Liver Dis 2012;44:477-81.

36. Hosoe N, Rey JF, Imaeda H, et al. Evaluations of capsule endoscopy software in reducing the reading time and the rate of false negatives by inexperienced endoscopists. Clin Res Hepatol Gastroenterol 2012;36:66-71.

37. Günther U, Daum S, Zeitz M, et al. Capsule endoscopy: comparison of two different reading modes. Int J Colorectal Dis 2012;27:521-5.

38. Westerhof J, Koornstra JJ, Weersma RK. Can we reduce capsule endoscopy reading times? Gastrointest Endosc 2009;69:497-502.

39. Koulaouzidis A, Rondonotti E, Karargyris A. Small-bowel capsule endoscopy: a ten-point contemporary review. World J Gastroenterol 2013;19:3726-46.

40. Koulaouzidis A, Smirnidis A, Douglas S, et al. QuickView in small-bowel capsule endoscopy is useful in certain clinical settings, but QuickView with Blue Mode is of no additional benefit. Eur J Gastroenterol Hepatol 2012;24:1099-104.

41. Liangpunsakul S, Mays L, Rex DK. Performance of Given suspected blood indicator. Am J Gastroenterol 2003;98:2676-8.

42. Buscaglia JM, Giday SA, Kantsevoy SV, et al. Performance characteristics of the suspected blood indicator feature in capsule endoscopy according to indication for study. Clin Gastroenterol Hepatol 2008;6:298-301.

43. D'Halluin PN, Delvaux M, Lapalus MG, et al. Does the "Suspected Blood Indicator" improve the detection of bleeding lesions by capsule endoscopy? Gastrointest Endosc 2005;61:243-9.
44. Iakovidis DK, Koulaouzidis A. Software for enhanced video capsule endoscopy: challenges for essential progress. Nat Rev Gastroenterol Hepatol 2015;12:172-86.

45. Iakovidis DK, Koulaouzidis A. Automatic lesion detection in capsule endoscopy based on color saliency: closer to an essential adjunct for reviewing software. Gastrointest Endosc 2014;80:877-83.

46. Iakovidis DK, Sarmiento R, Silva JS, et al. Towards intelligent capsules for robust wireless endoscopic imaging of the gut. IEEE International Conference on Imaging Systems and Techniques. Santorini, Greece, 2014:95-100.

47. Koulaouzidis A, Iakovidis DK. KID: A capsule endoscopy database for medical decision support. United European Gastroenterology Week (UEGW). Barcelona, Spain, 2015.

48. Spada C, Hassan C, Costamagna G. Virtual chromoendoscopy: will it play a role in capsule endoscopy? Dig Liver Dis 2011;43:927-8.

49. Rimbaş M, Negreanu L, Ciobanu L, et al. Is virtual chromoendoscopy useful in the evaluation of subtle ulcerative small-bowel lesions detected by video capsule endoscopy? Endosc Int Open 2015;3:E615-20.

50. ASGE Technology Committee, Manfredi MA, Abu Dayyeh BK, et al. Electronic chromoendoscopy. Gastrointest Endosc 2015;81:249-61.

51. Abdelaal UM, Morita E, Nouda S, et al. Blue mode imaging may improve the detection and visualization of small-bowel lesions: A capsule endoscopy study. Saudi J Gastroenterol 2015;21:418-22.

52. Koulaouzidis A, Douglas S, Plevris JN. Blue mode does not offer any benefit over white light when calculating Lewis score in small-bowel capsule endoscopy. World J Gastrointest Endosc 2012;4:33-7.

53. Ryu CB, Song JY, Lee MS, et al. Does Capsule Endoscopy With Alice Improves Visibility of Small Bowel Lesions? Gastrointest Endosc 2013;77:AB466.

54. Aihara H, Ikeda K, Tajiri H. Image-enhanced capsule endoscopy based on the diagnosis of vascularity when using a new type of capsule. Gastrointest Endosc 2011;73:1274-9.

55. Keuchel M, Kurniawan N, Baltes P, et al. Quantitative measurements in capsule endoscopy. Comput Biol Med 2015;65:333-47.

56. Marya N, Karellas A, Foley A, et al. Computerized 3-dimensional localization of a video capsule in the abdominal cavity: validation by digital radiography. Gastrointest Endosc 2014;79:669-74.

57. Karargyris A, Koulaouzidis A. OdoCapsule: nextgeneration wireless capsule endoscopy with accurate lesion localization and video stabilization capabilities. IEEE 
Trans Biomed Eng 2015;62:352-60.

58. Than TD, Alici G, Zhou H, et al. A review of localization systems for robotic endoscopic capsules. IEEE Trans Biomed Eng 2012;59:2387-99.

59. Sidhu R, Sakellariou P, McAlindon ME, et al. Is formal training necessary for capsule endoscopy? The largest gastroenterology trainee study with controls. Dig Liver Dis 2008;40:298-302.

60. Niv Y, Niv G. Capsule endoscopy examination--preliminary review by a nurse. Dig Dis Sci 2005;50:2121-4.

61. Caunedo Alvarez A, García-Montes JM, Herrerías JM. Capsule endoscopy reviewed by a nurse: is it here to stay? Dig Liver Dis 2006;38:603-4.

Cite this article as: McAlindon ME, Ching HL, Yung D, Sidhu R, Koulaouzidis A. Capsule endoscopy of the small bowel. Ann Transl Med 2016;4(19):369. doi: 10.21037/atm.2016.09.18
62. Albert JG, Humbla O, McAlindon ME, et al. A Simple Evaluation Tool (ET-CET) Indicates Increase of Diagnostic Skills From Small Bowel Capsule Endoscopy Training Courses: A Prospective Observational European Multicenter Study. Medicine (Baltimore) 2015;94:e1941.

63. Rajan E, Iyer PG, Oxentenko AS, et al. Training in small-bowel capsule endoscopy: assessing and defining competency. Gastrointest Endosc 2013;78:617-22.

64. Sidhu R, Sanders DS, Kapur K, et al. Capsule endoscopy: is there a role for nurses as physician extenders? Gastroenterol Nurs 2007;30:45-8.

65. Iddan G, Meron G, Glukhovsky A, et al. Wireless capsule endoscopy. Nature 2000;405:417. 